\title{
A signal detection theory analysis of an unconscious perception effect
}

\author{
STEVEN J. HAASE \\ Gordon College, Barnesville, Georgia \\ and \\ JOHN THEIOS and RICK JENISON \\ University of Wisconsin, Madison, Wisconsin
}

\begin{abstract}
The independent observation model (Macmillan \& Creelman, 1991) is fitted to detection-identification data collected under conditions of heavy masking. The model accurately predicts a quantitative relationship between stimulus detection and stimulus identification over a wide range of detection performance. This model can also be used to offer a signal detection interpretation of the common finding of above-chance identification following a missed signal. While our finding is not a new one, the stimuli used in this experiment (redundant three-letter strings) differ slightly from those used in traditional signal detection work. Also, the stimuli were presented very briefly and heavily masked, conditions typical in the study of unconscious perception effects.
\end{abstract}

Many researchers have investigated the relationship between stimulus detection and stimulus identification (e.g., Benzschawel \& Cohn, 1985; Green, Weber, \& Duncan, 1977; Macmillan \& Creelman, 1991; Swensson \& Judy, 1981). The task used in these and other similar studies is called concurrent detection and identification. In a typical version of this task, an observer makes two responses regarding a single stimulus event (or single trial). The first response is a yes/no (or rating scale) stimulus detection task, where the observer makes a decision as to whether signal + noise was presented or noise alone. Next, the observer is forced to decide, among two or more alternatives, which target stimulus may have been presented (i.e., simple identification; e.g., "if a tone was presented, was it a high or low tone?').

Starr, Metz, Lusted, and Goodenough (1975) have shown that it is possible to derive a quantitative relationship between detection and identification using signal detection theory, and it has been shown (Benzschawel \& Cohn, 1985; Green et al., 1977; Starr et al., 1975) that this quantitative relationship provides a good description of the results of experiments involving a small number of stimuli (two to four). Moreover, the theory predicts that observers

This article is based on a portion of S.J.H.'s doctoral dissertation. This research was presented at the 64th Annual Meeting of the Midwestern Psychological Association, Chicago, May 7, 1994, and at the 35th Annual Meeting of the Psychonomic Society, St. Louis, November 12, 1994. The authors thank Tom Carr, Doug Creelman, Tony Greenwald, Neil Macmillan, Phil Merikle, Bruce Schneider, and two anonymous reviewers for valuable comments on earlier versions of this manuscript. Correspondence should be sent to S. J. Haase, who is now at the Department of Rehabilitation Medicine, University of Wisconsin, 1300 University Ave., Madison, WI 53706 (e-mail: shaase@facstaff.wisc.edu).

-Accepted by previous editor, Myron L. Braunstein can identify stimuli at an above-chance performance level even when they "miss" the stimuli, a result that has been reported in several experiments (Benzschawel \& Cohn, 1985; Green et al., 1977; Lindner, 1968; Marcel, 1983; Merikle \& Reingold, 1990; Rollman \& Nachmias, 1972). Often, such results (above-chance identification of stimuli that are "not detected") are interpreted as indicating unconscious perception (Marcel, 1983; Merikle \& Reingold, 1990).

According to contemporary theories of signal detectability (Green \& Swets, 1966), the distribution of sensory states is assumed to be continuous, and a sensory threshold is not assumed to exist. In Gaussian signal detection theory, it is assumed that the sensory events evoked by each level of the stimulus are normally distributed with equal variance. In a yes/no task, the observer is assumed to divide the sensory space into two mutually exclusive regions and to respond "yes, a signal is present" if a trial produces a result in one of the regions and "no, a signal is not present" if a trial produces a result in the other region. Note that there is no necessary relationship between consciousness and the boundary (criterion) used to define the "yes" and "no" regions. Thus, a "no" response does not necessarily mean that there was no conscious perception of the stimulus, and a "yes" response does not necessarily mean that the stimulus was consciously perceived (Macmillan \& Creelman, 1991).

To see how an observer could correctly identify a stimulus when it is not detected, consider a model of sensory activation where each stimulus evokes a response along two orthogonal sensory channels. Figure 1 depicts equal probability density contours for two stimuli along with the contour corresponding to "no stimulus." Even though in Figure 1, two of the circles are not overlapping, it should be noted that the three bivariate distributions are contin- 


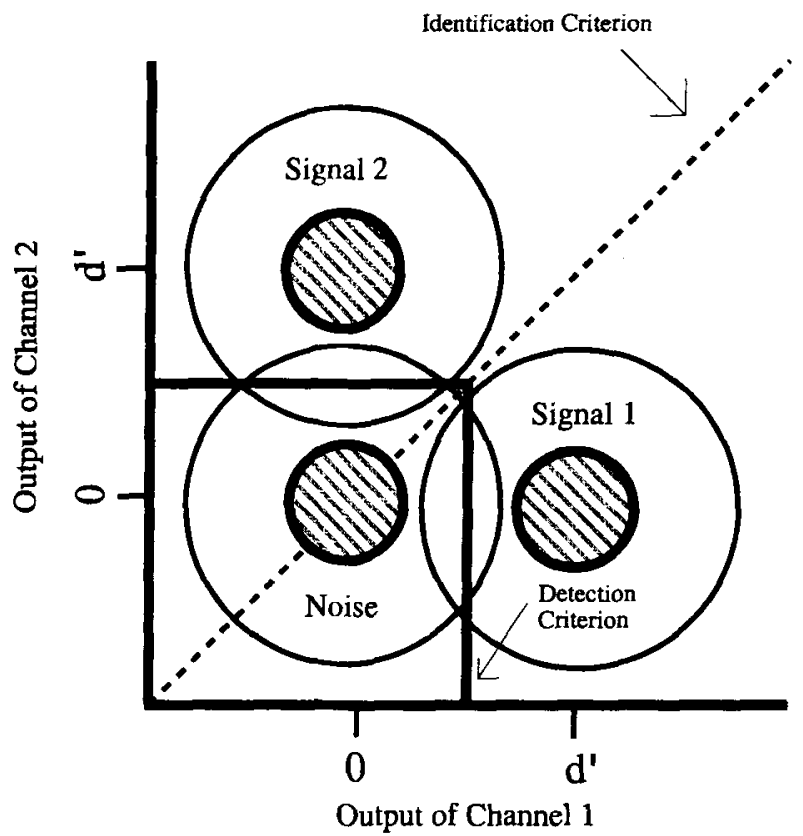

Figure 1. Detection-identification decision space. Note that, on detection miss trials, identification will be above chance. See text for details. Adapted from Detection Theory: $A$ User's Guide (p. 254), by N. A. Macmillan and C. D. Creelman, 1991, Cambridge: Cambridge University Press. Copyright 1991 by Cambridge University Press. Adapted with permission.

uous and overlap each other to some (perhaps small) degree everywhere. The noise distribution is represented in the bottom left corner of the figure. The observer is assumed to adopt two independent criteria when attempting to maximize performance on both tasks (Klein, 1985). The identification criterion is the line oriented at a $45^{\circ}$ angle. This criterion separates responses into "Target 1" classifications and "Target 2" classifications (simple identification). On each trial, the observer chooses the identification response based on whichever "channel" (Dimension 1 or Dimension 2) has the larger output (Macmillan \& Creelman, 1991; Swensson \& Judy, 1981). This level of sensory activity is also compared with the detection criterion for that trial. If the level of sensory activation falls above the detection criterion along either dimension (i.e., the solid square in the bottom left), the observer responds "yes, signal present." An observer responds nearly optimally by using this likelihood ratio detection rule (Klein, 1985). If the "no" region were to be identified as a state of consciousness in which the observer was unaware of the stimulus, then one could argue that identification could be above chance when the observer was unaware of the stimulus.

Marcel (1983), in using signal detection tasks as a tool to study unconscious perception, has argued that unconscious perception effects can be obtained even when detection performance is at chance. Although this claim has been difficult to verify empirically (see Holender, 1986), the detection model in Figure 1 makes a specific predic- tion about what should happen in such a situation. In this model, if detection performance is near chance (i.e., $d^{\prime}$ near zero), then identification performance would also be near chance (i.e., identification performance near .50; Green et al., 1977). Visually, the two-dimensional space would contain three completely overlapping distributions. In such a space, $d^{\prime}$ for stimulus detection would be near zero since there would be no separation between the noise-alone distribution and the two signal + noise distributions. Similarly, identification performance would be near chance since the two signal + noise distributions, would also be nearly, if not completely, overlapping.

The following experiment replicated the basic finding of above-chance identification performance following stimulus detection miss trials and also adopted a methodology that allowed application of Starr et al.'s (1975) recognition theorem. The unique aspects of this experiment, compared with earlier signal detection work (e.g., Benzschawel \& Cohn, 1985; Starr et al., 1975), were that the target stimuli were heavily masked and presented at very brief durations. In addition, the target stimuli used in this study (redundant letter strings) differed from those used in previous signal detection work.

\section{METHOD}

\section{Observers}

Fifteen students from the introductory psychology subject pool at the University of Wisconsin-Madison participated in this experiment in return for extra credit toward improving their course grade. All had normal or corrected-to-normal vision as measured by a Snellen chart. Twelve observers contributed data for analysis. The data from 1 observer were not included due to a computer error. The data from another observer were not included because of a zero hit rate and an extremely conservative response bias. The data of a 3rd observer were not analyzed because he did not complete the second and third session. The 11 females and 1 male ranged in age from 17 to 19 years.

\section{Materials and Apparatus}

The Micro Experimental Laboratory (MEL) software system (Schneider, 1990) controlled stimulus presentations and collected observers' responses. Stimuli were displayed on an IBM-PC compatible computer with an NEC-MultiSync monitor equipped with P-31 phosphor. The display decayed to about $37 \%$ of its original intensity after about $1.5 \mathrm{msec}$ and to less than $1 \%$ of its original intensity after about $3 \mathrm{msec}$. The timing of displays was verified by a digital oscilloscope. Target stimuli consisted of two three-letter strings (CCC or ZZZ). Pilot testing revealed these two strings to be roughly equally detectable, and previous research using confusion matrices has shown them to be orthogonal (not at all confusable), at least when each target letter was presented as a singleton (Townsend, 1971, Condition 2, poststimulus noise field). The masking stimuli consisted of different random arrangements (from trial to trial) of three symbolic characters ( 0 , if, and \#) from the ASCII character set that provided adequate masking and minimal confusability with the target letters. The only constraint on the selection of forward and backward mask was that a character did not repeat in the same position from forward to backward mask, resulting in six forward-backward mask combinations.

The observers viewed the stimuli at a distance of approximately $45 \mathrm{~cm}$. At this distance, each character in the display subtended approximately $0.40^{\circ}$ of horizontal visual angle and approximately 
$0.60^{\circ}$ of vertical visual angle. The three character target display subtended approximately $1.27^{\circ}$. The luminance of the display was $14 \mathrm{~cd} / \mathrm{m}^{2}$ when a uniform patch (white) was measured against the dark screen background used on the test trials.

\section{Procedure}

Each observer was tested individually in a moderately lit room (ambient light reflected off the dark screen was about $2 \mathrm{~cd} / \mathrm{m}^{2}$ ) over a period of 3 days. The 1 st day provided the observers with practice at detecting the targets. The 2 nd and 3 rd testing days comprised the concurrent detection-identification task. The concurrent detectionidentification trials differed from the detection practice trials in that the observers followed the detection response with a simple identification response. Also, detection-identification trials presented targets at the most difficult duration $(16.67 \mathrm{msec})$ used during the detection training session.

On the 1 st day, the experimenter read aloud instructions that were displayed on the monitor. The observers were instructed to read along and to ask questions if they had any. The observers were informed that the task would get successively more difficult after each block of trials, but, regardless of this, they should do their best even if they felt they were guessing. The experimenter remained in the room for a few practice trials to make sure the observers understood the instructions. The observers then proceeded through four blocks of detection trials. The exposure duration of the target field decreased successively across the four blocks of detection practice trials. Each target was presented an equal number of times in a pseudorandom order (sample without replacement) on $50 \%$ of the trials (signal + noise trials). The other trials presented masks only (noise trials). Also, signal + noise trials and noise-alone trials were presented in a pseudorandom order. See Table 1 for a general outline of the trial sequence (absent the identification task).

The target field was immediately followed by a backward mask for about $50 \mathrm{msec}$. The backward mask consisted of a different random arrangement of the characters in the forward mask for that trial. The stimulus-backward mask onset asynchrony was reduced across successive blocks as follows: Block 1, $133 \mathrm{msec}, 96$ trials; Block 2, 67 msec, 96 trials; Block 3, 33 msec, 144 trials; Block 4, $16.67 \mathrm{msec}, 144$ trials.

Following those events, which defined a single trial, the observers rated their confidence that a target letter string was presented. A 6-point detection rating scale was adopted: -3 (absolutely sure a target was not presented) to +3 (absolutely sure a target was presented). The zero or neutral point was absent, in order to force the observers to make "yes" or "no" detection decisions to varying degrees of certainty. The next trial began after a 500-msec intertrial interval. This first detection-only session took about $35 \mathrm{~min}$ for the observers to complete.

The experimental trials were carried out in a similar fashion and were completed in two sessions within 2 weeks of the detection practice session (see Table 1 for an outline of the trial sequence). The observers were again given instructions regarding the task and were

Table 1

Trial Sequence

\begin{tabular}{|c|c|c|}
\hline Event & Display & Duration (msec) \\
\hline Warning tone & blank & 500 \\
\hline Fixation & --- --- & 1,000 \\
\hline Forward mask & $0 \#$ & 50 \\
\hline Target display & $\mathrm{CCC}$ or $\mathrm{ZZZ}$ & 16.67 \\
\hline Backward mask & 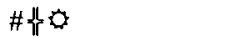 & 50 \\
\hline Detection rating & 6-point rating scale & until response \\
\hline $\begin{array}{l}\text { Identification response } \\
\text { (on experimental trials only) }\end{array}$ & $\mathrm{CCC}$ or $\mathrm{ZZZ}$ & until response \\
\hline Intertrial interval & blank & 500 \\
\hline
\end{tabular}

given the opportunity to have any of their questions answered. Each experimental session contained 960 trials (target duration $16.67 \mathrm{msec}$ ) presented in identical fashion to the practice trials, with one exception: After the observers gave their detection rating, they indicated which of the two stimuli may have been presented (an identification response). An identification response was made after every trial, regardless of whether or not a signal had been presented. The observers were told that, because this was a difficult task, there might be trials where a target would be presented even though they might express little confidence that such an event occurred. Thus, regardless of their detection response, they were asked to always give their best guess as to which target may have been presented. Once this was explained, the observers had little difficulty performing the task. Each experimental session took about $50 \mathrm{~min}$ for the observers to complete.

\section{RESULTS AND DISCUSSION}

Detection data were summarized using the tools of Gaussian signal detection theory (Green \& Swets, 1966; Macmillan \& Creelman, 1991). Where statistical tests were performed, we adopted the .05 level of significance; $p$ values are reported for the reader's convenience.

First, if we identify a "yes" response with confidence ratings of 1,2 , and 3 , and if we identify a "no" response with confidence ratings of $-3,-2$, and -1 , we can analyze the data as if it were a yes/no signal detection experiment and determine $d^{\prime}$ and the value of the criterion for each subject. These results are summarized in Table 2. All 12 observers were able to discriminate targets from blank fields (i.e., all $d^{\prime}$ s $>0$ ). A more detailed receiver operating characteristic (ROC) analysis will be presented shortly. Figure 2 graphs identification performance conditional on rating category. These data are averaged across the 12 observers. Each observer's mean identification performance was based on at least 15 observations. As can be seen clearly, the curve is monotonically increasing across the levels of detection confidence rating category. This observation is verified by the strong linear relationship between rating category and identification accuracy $\left(R^{2}=.97\right)$.

Next, as would be typical for an experiment on unconscious perception, identification performance was assessed, conditional on misses. For the -1 rating category (guessing that no stimulus was presented), identification performance $(M=.57)$ was reliably above chance $[t(11)$ $=3.08, S E=0.024, p<.01]$. For the -2 rating (think that no stimulus was presented), identification performance $(M=.55)$ was marginally above chance $[t(10)=1.42$, $S E=0.036, p<.10]$. Lastly, for the -3 rating (absolutely sure that no stimulus was presented), identification performance $(M=.51)$ was not reliably different from chance $[t(3)=0.18, S E=0.038, p>.40]$. Only 4 observers contributed data for the -3 rating category, whereas 12 and 11 observers contributed data for the -1 and -2 categories, respectively, which could decrease one's confidence that the -3 data point was reliable. Unless otherwise noted, tests of the above sort were conducted using a right-tailed rejection region (since the prediction is for above-chance identification performance). 
Table 2

Hit Rate (H), False-Alarm Rate (FA), Detectability ( $\left.d^{\prime}\right)$, and Criterion (c) Averaged Across the 12 Observers in Our Study

\begin{tabular}{llllr}
\hline & H & FA & $d^{\prime}$ & $c$ \\
\hline$M$ & .577 & .329 & .685 & +.139 \\
$S E$ & .038 & .043 & .154 & .086 \\
\hline
\end{tabular}

Note- $d^{\prime}=z(\mathrm{H})-z(\mathrm{FA}) ; c=-.5[z(\mathrm{H})+z(\mathrm{FA})] .0$, unbiased; + , conservative; - , liberal.

We also determined whether there were any differences between the combinations of characters used for masking. There were a total of six different forwardbackward masking combinations. A single-factor repeated measures analysis of variance (ANOVA) revealed that the main effect of masking combination was not significant $\left[F(5,55)=0.64, M S_{\mathrm{e}}=0.088, p>.60\right]$.

Essentially, this pattern of data replicates previous findings (e.g., Green et al., 1977; Merikle \& Reingold, 1990). The interpretation offered by Gaussian signal detection theory is straightforward: Stimuli that fall below the detection criterion may, nonetheless, be correctly identified at least on some of the trials (see Figure 1). Also, one need not assume that correct identifications following missed signals were based on unconscious perceptual processes.

Next, Starr et al's. (1975) recognition theorem was applied to the detection and identification data to determine whether detection and identification were quantitatively related. We consider the theorem in terms of $P\left(R^{\wedge} C \mid S\right)$, where $R$ is the rating category selected (from -3 to +3 ) and $C$ represents a correct identification re- sponse so that $P\left(R^{\wedge} C \mid S\right)$ refers to the probability of the joint occurrence of a rating and a correct identification response on a signal + noise trial (see Macmillan \& Creelman, 1991). In our task, some of the ratings $(-1,-2$, and -3) represented "no" detection responses, at least conceptually. This notation differs slightly from the notation used by Starr et al. (1975) and Green et al. (1977), but the mechanics of the theorem are unchanged.

The recognition theorem was applied to the multipoint ROC detection data generated in the present experiment. ${ }^{1}$ Figure 3 shows three operating characteristic curves. The top ROC curve plots the group averaged detection data. The curve below, in this plot, is the predicted identification operating characteristic (IOC; see Macmillan \& Creelman, 1991). Also plotted are the actual identification data with which to compare the predicted values. This plot, the bottom curve in Figure 3, indicates that, in general, the observed identification data fell near the predicted values, but systematically below. Overall, the average absolute error of this parameter-free prediction was .04 , which is close to the values obtained in the Green et al. (1977) study. The small discrepancy in prediction was, in part, due to the propagation of error across cumulative data points.

How does this model relate to unconscious perception? To answer this question, the data need to be separated from the model. In Figure 3, the data represent cumulative proportions starting at the bottom left with a conservative detection criterion and moving to the upper right with more liberal detection criteria. At the most conservative

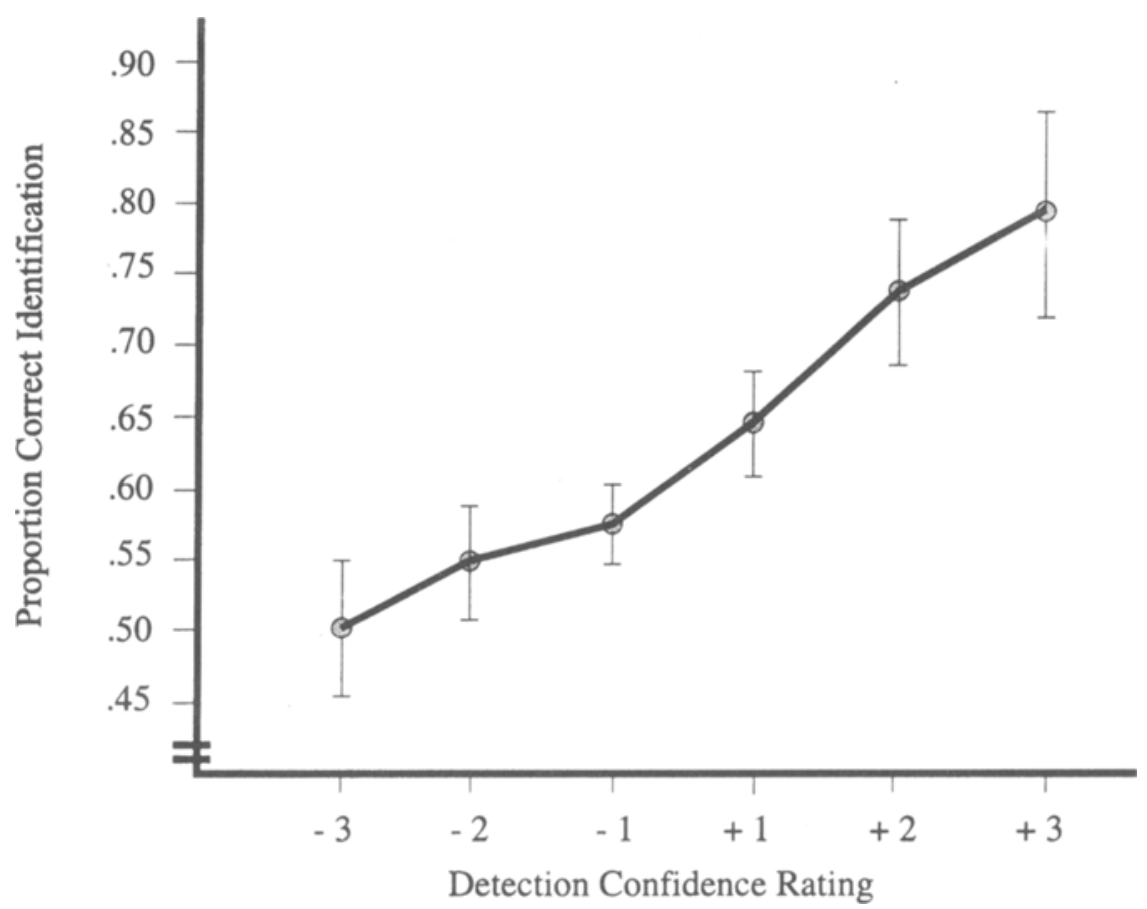

Figure 2. Stimulus identification performance conditional on detection rating category selected. On "miss" trials identification was above chance at the -1 (guessing that no stimulus was presented) rating category. 


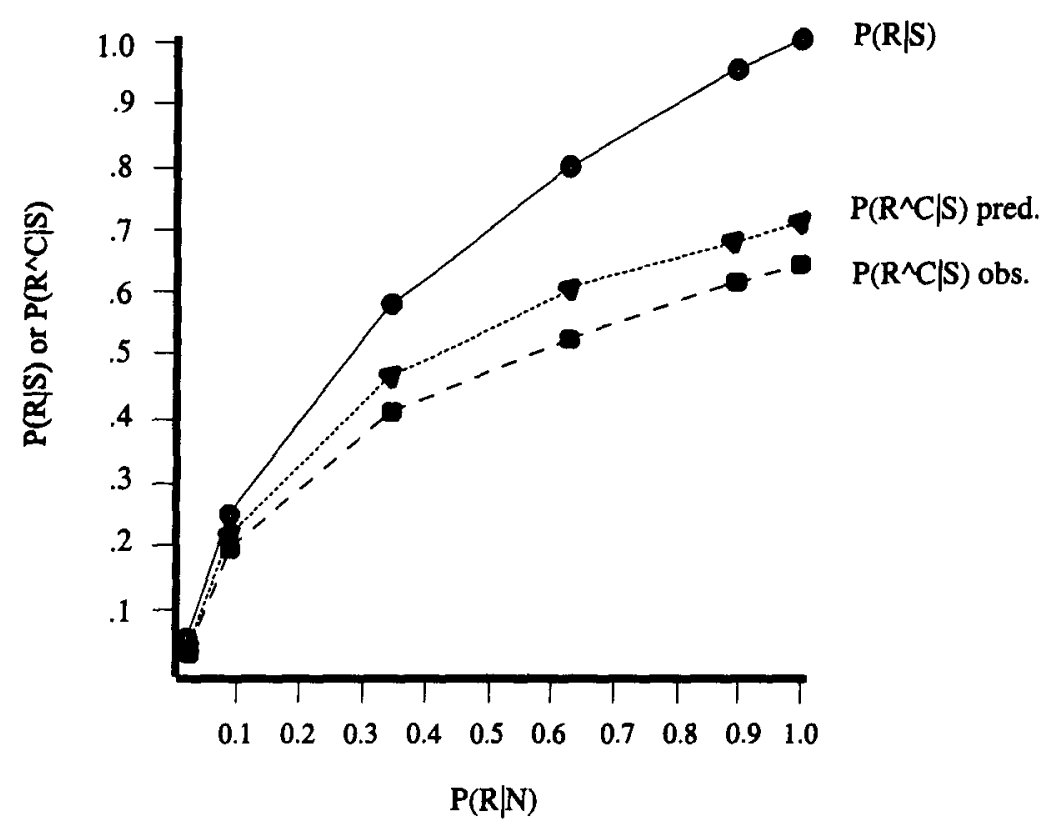

\begin{abstract}
Figure 3. Receiver operating characteristic for detection (top curve), predicted identification operating characteristic (IOC, middle curve), and observed IOC (lower curve). Data are averaged across the 12 observers from Experiment 1. These data are cumulative proportions; however, if one examines the relative proportions at each criterion, identification performance is above chance even when observers "missed" the signal (see Figure 2). However, this is predicted from the model (see Figure 1 and text explanation). Note that $P\left(R^{\wedge} C \mid S\right)$ reads as the probability of the detection rating category and correct stimulus identification given signal + noise trial.
\end{abstract}

detection criterion, the model predicts that identification performance should be very accurate. This was indeed the case, given that the proportion of targets correctly identified using this criterion $(+3)$ was about $88 \%$. Concerning miss trials, one would have to examine the noncumulative proportions from the more "lenient" criteria $(-1,-2$, and -3$)$. When an observation fell within the criterion range -3 , the observers' identification performance on signal + noise trials was about $46 \%$. Performance exceeded chance slightly at the next criterion $(-2)$ at $53 \%$. At the -1 criterion, identification performance was $56 \%$. These figures agree, for the most part, with the data in Figure 2, although they were computed slightly differently here (a group average in the ROC/IOC rather than an average of individual observer averages in the case of Figure 2). The point is that this model predicts abovechance performance even when observers "miss" the signal. As mentioned, performance was lower than predicted, possibly due to the fact that the $\mathrm{ZZZ}$ target string appeared to be easier to detect $\left(d^{\prime}=.766, S E=.146\right)$ than the CCC string $\left(d^{\prime}=.619, S E=.196\right)$. This difference, however, was not statistically significant $[t(11)=$ $1.18, p>.20$, two-tailed dependent samples $t$ test]. Again, since the model assumes that targets are equally detectable, such a violation would result in less than perfect prediction. This effect can also be observed in a $z$-score plot of the ROC. Figure 4 shows a linear plot as predicted by Gaussian signal detection theory. When the distributions of noise alone and signal + noise have equal variances, the plot has a slope of 1 . The slope being slightly less than 1 in this plot (slope $=.86$ ) indicates that the variance of the signal + noise distribution is larger than the variance of the noise-alone distribution (Macmillan \& Creelman, 1991). Since the ZZZ string was (marginally) easier to detect than the CCC string, the signal + noise distribution contains a weak signal and a slightly stronger signal resulting in a more variable distribution.

In terms of conscious versus unconscious perception, the results of our experiment are consistent with several interpretations, one of them being a continuum of awareness. The question remains as to whether or not it is useful to conclude that the ability of an observer to correctly identify a missed target is an indication of unconscious perception and, by default, that a correctly detected stimulus is thus consciously identified.

The geometric decision space of the independent observation model (Figure 1) shows that a stimulus falling below the detection criterion can nonetheless be identified at least some of the time. In other words, the distinction between conscious and unconscious perception is not needed to account for these data. Also, there is no point in this model where one could distinguish between 


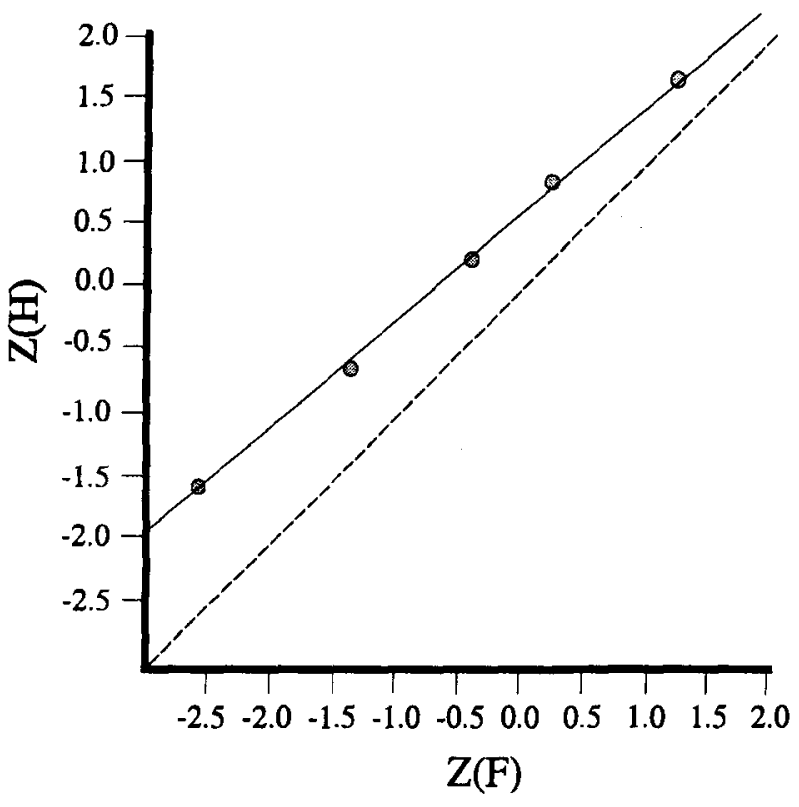

Figure 4. ROC line plotted in $z$-score coordinates.

conscious and unconscious perception. That is, the relationship between detection and identification is quantitative rather than qualitative.

It is noted that, subjectively, observers often show little confidence in their identification responses when they believe that no stimulus was presented. When they correctly identify these missed stimuli, the question remains as to whether correct identification was based on unconscious perceptual processes or perhaps a vague awareness that the target + noise field was more similar to one target than another (i.e., sophisticated guessing). These subjective factors need not be assumed as explaining nor affecting the results of our study. These results fit with a decision process model that does not need conscious, unconscious, or a combination of consciousunconscious processes added to it.

\section{GENERAL DISCUSSION}

The implications of the present work are that the independent observation model (Macmillan \& Creelman, 1991) presented in Figure 1 and the recognition theorem of Starr et al. (1975) adequately account for the relationship between stimulus detection and stimulus identification. The data support Gaussian signal detection theory's claim that sensory states are continuous in that, as detection accuracy increases, so does identification accuracy. These findings have been documented in other psychophysical experiments investigating the detection and localization of malignancy in radiographic images (Starr et al., 1975), detection and identification of auditory tones (Green et al., 1977), and gap detection and identification in Landolt Cs (Benzschawel \& Cohn, 1985). The purpose of these earlier experiments, however, was not to address issues related to conscious versus unconscious perception. Also, the stimuli in our study were presented for much briefer durations, under conditions of heavy visual masking, typical in studies of "unconscious perception."

Further questions that need to be addressed in empirical research are whether the present framework can be applied to larger, more meaningful stimulus sets, such as the ones Merikle and Reingold (1990) used. Macmillan (1986) suggests that the independent observation model should hold for lexical and pictorial stimuli (in addition to tones, simple visual displays, etc.) if these more complex stimuli are represented in an orthogonal decision space. Others might question the application of Gaussian signal detection theory to such stimulus sets and whether, for example, the recognition theorem would necessarily be expected to hold since it may be difficult to specify the probability distributions for such unconstrained stimulus sets (Robert Lutfi, personal communication, May 13, 1994). Previous research by Green et al. suggests that the observer's task becomes very difficult when identification is based on one of four possible signals. Perhaps the model could be adapted to a situation such as Merikle and Reingold's (1990) word detection-identification experiments, in which the observer's identification response is based on a choice from two possible signals, even though the pool of potential signals is much larger. Another interesting issue that could be explored is whether a signal detection model could account for the qualitative differences Merikle and Reingold found for words versus nonwords. In their study, above-chance identification following detection misses occurred only for word stimuli. They argued that the dissociation between detection and identification will be obtained only when the stimuli are familiar and have representations in memory that can be unconsciously activated.

\section{REFERENCES}

Benzschawel, T., \& CoHn, T. E. (1985). Detection and recognition of visual targets. Journal of the Optical Society of America A, 2, 1543-1550.

Green, D. M., \& Swets, J. A. (1966). Signal detection theory and psychophysics. New York: Wiley.

GreEn, D. M., Weber, D. L., \& Duncan, J. E. (1977). Detection and recognition of pure tones in noise. Journal of the Acoustical Society of America, 62, 948-954.

HAASE, S. J. (1994). A signal detection theory interpretation of "unconscious" perception (Doctoral dissertation, University of WisconsinMadison, 1994). Dissertation Abstracts International, 55 (06), 2422B.

HolendER, D. (1986). Semantic activation without conscious identification in dichotic listening, parafoveal vision, and visual masking: A survey and appraisal. Behavioral \& Brain Sciences, 9, 1-66.

KLEIN, S. A. (1985). Double-judgment psychophysics: Problems and solutions. Journal of the Optical Society of America A, 2, 1560-1585.

LINDNER, W. A. (1968). Recognition performance as a function of detection criterion in a simultaneous detection-recognition task. Journal of the Acoustical Society of America, 44, 204-211.

MaCMillaN, N. A. (1986). The psychophysics of subliminal perception. Behavioral \& Brain Sciences, 9, 38-39.

Macmillan, N. A., \& Creelman, C. D. (1991). Detection theory: A user's guide. Cambridge: Cambridge University Press.

MaCmillan, N. A., \& KaPlan, H. L. (1985). Detection theory analysis 
of group data: Estimating sensitivity from average hit and false-alarm rates. Psychological Bulletin, 98, 185-199.

MarCEL, A. J. (1983). Conscious and unconscious perception: Experiments on visual masking and word recognition. Cognitive Psychology, 15, 197-237.

Merikle, P. M., \& Reingold, E. M. (1990). Recognition and lexical decision without detection: Unconscious perception? Journal of Experimental Psychology: Human Perception \& Performance, 16, 574-583.

Rollman, G. B., \& NaChmias, J. (1972). Simultaneous detection and recognition of chromatic flashes. Perception \& Psychophysics, 12, 309-314.

SCHNEIDER, W. (1990). Micro Experimental Laboratory [Computer software]. Pittsburgh: Psychology Software Tools.

Stark, S. J., Metz, C. E., Lusted, L. B., \& Goodenough, D. J. (1975). Visual detection and localization of radiographic images. Radiology, 116, 533-538.

SWENSSON, R. G., \& JUDY, P. F. (1981). Detection of noisy visual targets: Models for the effects of spatial uncertainty and signal-to-noise ratio. Perception \& Psychophysics, 29, 521- 534.

TOWNSEND, J. T. (1971). Theoretical analysis of an alphabetic confusion matrix. Perception \& Psychophysics, 9, 40-50.

\section{NOTE}

1. Macmillan and Kaplan (1985) suggest that averaging the raw proportions of hit and false-alarm rates will not yield a $d^{\prime}$ estimate much different from computing $d^{\prime}$ from each observer and then averaging these $d^{\prime}$ scores to obtain an average $d^{\prime}$. Computing individual $d^{\prime}$ and then averaging does yield a more accurate measure of group sensitivity if there are substantial differences in bias across observers. A similar argument would apply to constructing an average ROC curve, as we have presented here. Individual observer ROCs were plotted for those observers who used each rating category with enough frequency to construct a multipoint ROC. These individual observer figures were very similar in appearance to the group ROC. Importantly, the IOC for each observer was very close to the predicted values, as was the case for the group-averaged data (Haase, 1994).

(Manuscript received December 20, 1996; revision accepted for publication June 6, 1998.) 